\title{
Desmame precoce entre lactentes em Hospital Filantrópico de Aracaju
}

O estudo em análise tem como objetivo avaliar as causas mais prevalentes do desmame precoce nos recém-nascidos e lactentes atendidos em Urgência Pediátrica do Hospital Filantrópico no período de janeiro a abril de 2019 e estimular o auxílio dos profissionais de saúde para que as dificuldades maternas em relação ao aleitamento materno sejam sanadas. Trata-se de um estudo transversal, prospectivo e analítico realizado através da aplicação de questionário, formulado com dados relevantes da literatura, aos acompanhantes dos recém-nascidos e lactentes de 0 a 12 meses atendidos na Urgência Pediátrica. Foi visto que o aleitamento materno exclusivo em menores de 6 meses foi de 35,6\% ( $n=26)$ e de aleitamento predominante $15,1 \%$ ( $n=11$ ). 0 tempo médio de amamentação exclusiva foi de aproximadamente 2 meses. Dentre os motivos de desmame precoce, $51 \%(n=46)$ das mães referiram que deixaram de amamentar pelo mito do leite ser fraco ou por ter pouca quantidade; $14 \%(n=13)$ por conta da vida profissional e $14 \%$ ( $n=13$ ) relata que a criança perdeu o interesse. Diante da pesquisa trazida, pôde-se concluir que os principais motivos do desmame precoce se relacionam com a quantidade e qualidade do leite materno seguido de retorno ao trabalho e o lactente perder o interesse pelo aleitamento.

Palavras-chave: Aleitamento materno; Desmame precoce; Prevalência.

\section{Early weaning among infants in Philanthropic Hospital in Aracaju}

The objective of the present study is to assess the most prevalent causes of early weaning in infants attended in Pediatric Emergency at the Philanthropic Hospital from January to April 2019 and to encourage the help of health professionals, so maternal difficulties in relation to breastfeeding can be resolved. It is a cross-sectional, analytical study, based on data collected from questionnaires formulated through relevant information sourced from high-quality published research, to the mothers of infants aged 0 to 12 months who were being treated at the pediatric urgent care. Was spotted that exclusive breastfeeding in infants under 6 months was 35,6\% $(n=26)$, while predominant breastfeeding was $15,1 \%(n=11)$. The average period of time during which the infants had been exclusively breastfed was 2 months. With respect to early weaning, $51 \%(n=46)$ of mothers reported milk being weak or low in quantity as the reason; $14 \%(n=$ 13) attributed it to their professional careers and $14 \%(n=13)$ reported a loss of interest by the infant. In view of this research, it was concluded found that the main reasons for early weaning are related to the quantity and quality of breast milk, followed by returning to work and the infant losing interest in breastfeeding.

Keywords: Breastfeeding; Early weaning; Prevalence.

Topic: Ciências Sociais e Humanas em Saúde

Reviewed anonymously in the process of blind peer.
Received: 25/04/2021

Approved: 15/05/2021
Naiana Mota Araujo (D)

Universidade Tiradentes, Brasil

http://lattes.cnpq.br/7869745620432889

http://orcid.org/0000-0002-6908-1713

naianamota@hotmail.com

Adriana Dantas Lopes (iD)

Universidade Tiradentes, Brasil

http://lattes.cnpq.br/8509760460392344

http://orcid.org/0000-0002-6523-7442

adrianadantaslopes@yahoo.com.br

Annye Beatriz Cabral Santana (ib)

Universidade Federal de Sergipe, Brasil

http://lattes.cnpq.br/7541355556489269

http://orcid.org/0000-0002-8972-9855

annyebeatriz1997@gmail.com
Gabriel Dantas Lopes (D)

Universidade Tiradentes, Brasil

http://lattes.cnpq.br/1412495355733136

http://orcid.org/0000-0002-9743-6825

skiperlopes@gmail.com

Gabriella Mello Rusciolelli Nunes (iD)

Universidade Federal de Sergipe, Brasil

http://lattes.cnpq.br/4082562262510588

http://orcid.org/0000-0002-2177-2785

gabby_rusciolelli@hotmail.com

Izailza Matos Dantas Lopes (it)

Universidade Tiradentes, Brasil

http://lattes.cnpq.br/5911269328493167

http://orcid.org/0000-0001-9752-5628

Izailza.matos@souunit.com.br
Yasmim Laila Fragoso Cestari (id

Universidade Tiradentes, Brasil

http://lattes.cnpq.br/8069587594568547

http://orcid.org/0000-0001-6363-0542

yasmim.laila@souunit.com.br

\section{Referencing this:}

ARAUJO, N. M.; LOPES, A. D.; SANTANA, A. B. C.; LOPES, G. D.; NUNES, G. M. R; LOPES, I. M. D.; CESTARI, Y. L. F.. Desmame precoce entre lactentes em Hospital Filantrópico de Aracaju. Scire Salutis, v.11, n.2, p.163-170, 2021. DOI: http://doi.org/10.6008/CBPC2236$\underline{9600.2021 .002 .0019}$ 


\section{INTRODUÇÃO}

A prática do aleitamento materno é recomendada pela Organização Mundial da Saúde (OMS) e Ministério da saúde de forma exclusiva, ou seja, como único alimento, até os seis meses de vida do lactente. Esta idade considera a maturação fisiológica e neurológica que tornarão a criança apta a receber outros tipos de alimentos (PARIZOTTO et al., 2008).

A introdução de qualquer tipo de alimento na dieta de uma criança menor que seis meses de idade a qual se encontrava em Aleitamento Materno Exclusivo (AME) define o desmame precoce, que tem se tornado uma realidade no Brasil. Dados da última pesquisa nacional apontam que apenas $41 \%$ dos menores de seis meses no conjunto das capitais brasileiras e Distrito Federal estavam em AME, sendo a duração mediana do aleitamento materno exclusivo de 54,1 dias (1,8 meses). A Região Norte apresentou a maior prevalência $(45,9 \%)$, seguida da Centro-Oeste $(45,0 \%)$, Sul $(43,9 \%)$ e Sudeste $(39,4 \%)$, com a Nordeste apresentando a pior taxa (37,0\%) (VENANCIO et al., 2010).

As causas para o abandono do AME são diversas. Uma revisão sistemática feita em 2017 identificou por categorias algumas delas. Entre as características do recém-nascido associaram os fatores: o bebê recusa o peito ou não quer mais mamar $(12,8 \%)$, doença/hospitalização $(7,7 \%)$, baixo peso ao nascer $(7,7 \%)$, gemelaridade $(5,1 \%)$, bebê morde o peito $(2,6 \%)$ e ganho de peso insuficiente $(2,6 \%)$. Quanto à categoria que constam causas maternas, tem-se trabalho (33,3\%), escolaridade materna/paterna $(15,4 \%)$, renda familiar baixa $(12,8 \%)$, idade materna $(10,2 \%)$, tabagismo $(7,7 \%)$, tipo de parto $(5,1 \%)$, decisão materna $(5,1 \%)$, depressão $(5,1 \%)$ e paridade $(5,1 \%)$. Já a categoria relacionada aos mitos da amamentação tem o uso de chupeta $(35,9 \%)$, leite fraco $(17,9 \%)$, leite insuficiente ou pouco $(10,2 \%)$, leite secou $(5,1 \%)$ e choro do bebê- interpretado como fome $(5,1 \%)$. Foram, também, encontrados outros fatores prevalentes: trauma mamilar (17,9\%) e introdução de outros tipos de leite, com 15,4\% (ALVARENGA et al., 2017).

Nesse contexto, é imprescindível que sejam reconhecidas as principais causas do abandono do AME a fim de que sejam solucionadas e propiciem uma vida mais saudável para essas crianças (SANMAMED et al., 2013). Por conseguinte, o presente estudo objetiva identificar as principais causas do desmame precoce nos recém-nascidos e lactentes atendidos em Urgência Pediátrica do Hospital Filantrópico no período de janeiro a abril de 2019, além de estimular os profissionais de saúde a desmitificar mitos sobre a amamentação e promover a boa comunicação dos profissionais de saúde com a puérpera, auxiliando-a nas dificuldades que houver com o intuito de aumentar a prevalência do AME.

\section{METODOLOGIA}

Define-se por ser um estudo transversal, prospectivo e analítico. Para a quantidade da amostra total de pacientes adquirido, através da direção do Hospital Filantrópico foi obtido o quantitativo de 3.000 pacientes atendidos na Urgência Pediátrica em um mês. A pesquisa foi realizada em mães dos recémnascidos e lactentes de 0 a 12 meses, atendidos na urgência pediátrica de um hospital filantrópico no período de Janeiro a Abril de 2019. Considerando cálculos de nível de confiança, erro amostral e critérios utilizados de seleção e exclusão, foi considerado o n amostral de 131 crianças. Os dados foram coletados a 
partir de questionários compostos por questões de múltipla escolha de natureza clara e objetiva e perguntas restritas a uma opção como resposta.

Os critérios de seleção utilizados foram mães de recém-nascidos e lactentes de 0 a 12 meses e que concordaram em responder após a leitura e assinatura do Termo de Consentimento Livre e Esclarecido. Já os critérios de exclusão foram crianças além da faixa etária desejada, aquelas com escala de APGAR inferior a 7 pontos durante o 5o minuto de vida e/ou portadoras de doenças que resultam em dificuldades de sucção, o que proporcionaria um resultado inautêntico. Foram consideradas, ainda, tais variáveis maternas para o estudo: idade média, escolaridade, estado civil, renda familiar, paridade e cobertura por auxílio governamental, já do recém-nascido foram consideradas as seguintes: idade média, tipo de aleitamento recebido e uso de chupetas.

Os dados coletados foram armazenados em banco de dados padronizado através do software Excel versão 2015. Em seguida, analisados e interpretados utilizando a estatística descritiva. As variáveis categóricas foram apresentadas por meio de frequências simples e relativas e as variáveis numéricas descritas em média e desvio-padrão. Para comparação de médias de variáveis numéricas entre dois grupos, foi utilizado o teste t-Student para amostras independentes. O nível de significância estatística adotado foi de $5 \%(p \leq 0,05)$ e todos os testes foram bicaudais. O programa utilizado para as análises foi o Statistical Package for Social Sciences versão 21.0.

O estudo foi conduzido mantendo-se nos padrões exigidos pelo Comitê de Ética em Pesquisa com Seres Humanos da Universidade Tiradentes-CEP/UNIT, o qual foi aprovado no dia 10 de maio de 2019 com o CAAE/UNIT: 90598018.8 .0000 .5371$.

\section{RESULTADOS}

Participaram da pesquisa 131 recém-nascidos e lactentes entre 0 e 12 meses. Em relação às características maternas, a idade média foi de 25,6 anos, a escolaridade da maioria $(73,3 \%)$ foi de Ensino Fundamental completo, a taxa de fecundidade média foi de 2,2 e a renda familiar média foi de $\mathrm{R} \$ 1.072,27$, valor um pouco superior ao salário mínimo vigente (Tabela I).

Tabela I: Características maternas no Hospital do estudo de janeiro a abril de 2019.

\begin{tabular}{|l|l|l|}
\hline Mãe & $\mathbf{N}=\mathbf{1 3 1}$ & $\%$ \\
\hline Idade & & \\
$<18$ anos & 18 & 13,7 \\
$18-30$ anos & 79 & 60,3 \\
$31-40$ anos & 33 & 25,2 \\
$>40$ anos & 1 & 0,8 \\
\hline Escolaridade & & \\
\hline Até 8 anos & 40 & 29,8 \\
\hline Mais de 8 anos & 87 & 66,5 \\
\hline Ensino superior & 4 & 3,7 \\
\hline Estado civil & & \\
\hline Solteira & 33 & 25,2 \\
\hline Casada & 34 & 26,0 \\
\hline União estável & 64 & 48,8 \\
\hline Renda Familiar & & \\
\hline Menos de 1 salário-mínimo & 83 & 63,4 \\
\hline Entre 1 e 2 salários-mínimos & 36 & 27,4 \\
\hline Entre 2 e 3 salários-mínimos & 12 & 9,2 \\
\hline Paridade & & \\
\hline 1 & 46 & 35,1 \\
\hline
\end{tabular}




\begin{tabular}{|l|l|l|}
\hline 2 & 46 & 35,1 \\
\hline 3 a 4 & 31 & 23,6 \\
5 a 8 & 8 & 6,2 \\
Bolsa Família & 71 & 54,2 \\
Sim & 60 & 45,8 \\
Não & & \\
\hline
\end{tabular}

Quanto aos recém-nascidos e lactentes, foi observada uma média de idade de 5,2 meses, estando a maioria na faixa etária de até 1 mês de idade (Tabela II).

Tabela II: Idade dos recém-nascidos e lactentes no Hospital do estudo de janeiro a abril de 2019.

\begin{tabular}{|l|l|l|}
\hline Idade & $\mathbf{N}=\mathbf{1 3 1}$ & $\%$ \\
\hline $\mathbf{1}$ mês ou menos & 39 & 29,6 \\
\hline $\mathbf{1}$ a $\mathbf{3}$ meses & 17 & 13,1 \\
\hline a $\mathbf{5}$ meses & 17 & 13,1 \\
\hline $\mathbf{5}$ a $\mathbf{7}$ meses & 14 & 10,6 \\
\hline $\mathbf{7}$ a $\mathbf{m e s e s}$ & 17 & 13,1 \\
\hline $\mathbf{9}$ a $\mathbf{1 2}$ meses & $\mathbf{2 7}$ & $\mathbf{2 0 , 5}$ \\
\hline
\end{tabular}

Considerando apenas os lactentes e RN menores de 6 meses, o tipo de aleitamento mais prevalente foi o aleitamento materno exclusivo (AME), totalizando 26 lactentes. 0 aleitamento predominante foi observado em 11 lactentes, e 13 não recebiam leite materno (Figura 1). 0 tempo médio de amamentação exclusiva no presente estudo foi de aproximadamente 2 meses. Em relação ao uso da chupeta, 51,1\% dos lactentes faziam uso dela.

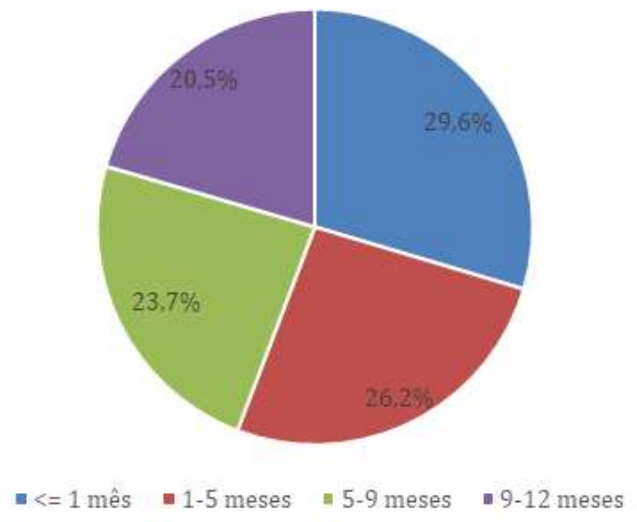

Figura 1: Idade dos Lactentes.

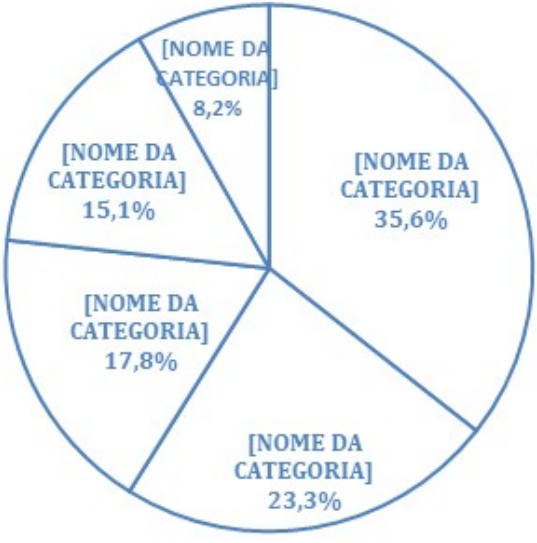

Figura 2: Alimentação atual dos menores de 6 meses.

Quanto aos motivos de desmame precoce, a maioria $(n=46)$ das mães referiu que deixou de amamentar por conta do mito do leite fraco ou por ter pouca quantidade, seguido pelas que disseram ter parado por conta da vida profissional $(n=13)$ e por perda de interesse da própria criança $(n=13)$. Outros motivos encontrados foram doença materna $(n=4)$, dor $(n=4)$, fissuras $(n=4)$, falta de apoio familiar $(n=2)$, mastite $(n=1)$, mamilo plano $(n=1)$, mamilo invertido $(n=1)$ e tabagismo ou alcoolismo $(n=1)$. (Figura 2$)$.

\section{DISCUSSÃO}

O desmame precoce é um fenômeno complexo, podendo ser influenciado por diversos fatores, como biológicos, culturais, socioeconômicos ou psíquicos, definindo-se um processo farto de ideologias e determinantes que resultam de condições inconscientes e concretas de vida (SAMPAIO et al., 2010).

Sabe-se que, embora a importância da amamentação em menores de 6 meses de idade seja notória, muitas mães optam pelo desmame. Isto pode ser observado no estudo em questão, no qual se 
constatou que o aleitamento materno exclusivo (AME) teve o tempo médio de 2 meses e a prevalência em menores de 6 meses de apenas 35,6\%.

Este fato também foi observado na Pesquisa Nacional de Saúde (PNS) dos anos 1986, 1996, 2006 e 2013, em que foi analisada a tendência dos indicadores de aleitamento materno no Brasil nas últimas três décadas. Demostrou-se que a prevalência do AME entre os menores de seis meses aumentou 34,2 pontos percentuais entre 1986 e 2006, indo de 2,9\% para 37,1\%, com ganhos estatisticamente significativos em cada década até 2006 e estabilização em 2013. No entanto, em 2013, houve redução da prevalência de AME de $0,3 \%$ entre os bebês de zero a dois meses de idade e 15,1 pontos percentuais entre os bebês de três a cinco meses de idade em relação ao inquérito de 2006 (BOCCOLINI et al., 2017). Por isso, constata-se a importância de se discutir a respeito das causas que levem ao desmame precoce.

O mito do leite fraco ou a pouca quantidade deste foi o principal problema encontrado na pesquisa, concentrando o motivo referido de 51\% das mães para deixarem de amamentar. Quanto ao primeiro mito, o estudo de Marques et al. (2011) ressaltou que não existe leite fraco, sendo essa crença pautada no desconhecimento das características inerentes do leite humano, o que leva a nutriz a desconfiar de sua capacidade de produzir leite de "qualidade". Foi feito, ainda, um comparativo com o trabalho de Escobar et al., o qual analisou as principais causas da complementação precoce relatadas pelas mães, sendo observado que $17,8 \%$ responderam que era devido ao seu "leite fraco" ou que seu leite "não sustentava" o bebê (MARQUES et al., 2011; ESCOBAR et al., 2002).

Com relação ao leite insuficiente, cabe destacar que estudos afirmam que a produção de leite materno pode diminuir quando: há a introdução da alimentação complementar com leite artificial, água ou chá, pois a criança vai perdendo o apetite; ocorre a introdução de chupetas e mamadeiras; ocorre pouca ingestão de líquidos e alimentação incorreta da mãe; nota-se despreparo da equipe de saúde em perceber posicionamento inadequado e pega incorreta - fato observado na mesma pesquisa, em que $5,1 \%$ das mães relataram não receber orientação sobre amamentação (ALVARENGA et al., 2017).

Barros et al. (1994) também discutem em seus estudos quanto ao tema do desmame por conta da qualidade do leite. Segundo eles, é muito comum que o desmame ocorra pelo fato de o bebê chorar muito - o que pressupõe uma atribuição, feita pela mãe e familiares, do choro à fome, concluindo que o leite materno pode ser insuficiente ou fraco e, por isso, a iniciação da alimentação com leite artificial (BARROS et al., 1994).

Em relação à utilização de chupetas, estudos de Cotrim et al. (2002) relatam que a chupeta pode influenciar negativamente o aleitamento materno, ocasionando confusão de bicos e diminuição da frequência das mamadas. Somado a isso, o trabalho relaciona este fato com as pesquisas de Victora et al., as quais relataram $65 \%$ de interrupção do $\mathrm{AM}$ aos seis meses em um grupo de crianças que usaram chupeta com um mês de idade, contra $24 \%$ no grupo que não usava (COTRIM et al., 2002; VICTORA et al., 1997). No presente estudo, $51 \%$ dos lactentes eram usuários de chupeta e a grande maioria iniciou o uso logo no primeiro mês de vida.

Outro empecilho ao aleitamento materno encontrado na pesquisa foi o retorno à vida profissional 
da mãe, totalizando $14,4 \%$ dos motivos relatados para o desmame precoce. Este fator também se mostrou prevalente na pesquisa realizada por Alvarenga et al. (2017), sendo relatado por 33,3\% das mães. Quanto a isso, Rea et al. (2002) afirmam que amamentar é um direito que a sociedade deve garantir a toda mulher e a toda criança. Assim, esse direito estende-se também à mulher que tem um trabalho remunerado (CARVALHO et al., 2002). Ademais, a lactante tem direito por lei de sair do local de trabalho duas vezes ao dia, por meia hora em cada vez, para amamentar. Caso essa alternativa não seja possível, a mãe tem o direito de retornar 1 hora mais cedo para a casa para amamentar (VINHA, 1999).

O estudo em questão também apontou que $14,4 \%$ das mães relataram que a criança perdeu o interesse na amamentação, fato que está em consonância à pesquisa de Alvarenga et al. (2017), em que $12,8 \%$ das mães afirmaram que realizaram o desmame precoce por conta de o bebê recusar o peito ou não querer mais mamar.

A recusa do bebê ao peito pode ocorrer devido a uma má postura ou causas físicas, como: o bebê possuir uma boca demasiado pequena ou o peito ser grande porque a aréola está tensa e faz com que o peito fique mais plano; os bebês imaturos que ainda não desenvolveram o reflexo de sucção; ou algumas iatrogenias decorridas do parto, como luxação de ombros, que produz dor no bebê pela posição e, consequentemente, faz com que não queira mamar (ALVARENGA et al., 2017).

Problemas mamários também foram relatados na pesquisa, tais como: dor $(4,5 \%)$, fissuras $(4,5 \%)$, mamilo plano ou invertido $(2,1 \%)$ e mastite $(1,1 \%)$. Estes dados também foram avaliados no estudo de Oliveira et al. (2015), em que participaram mulheres que tiveram filhos de janeiro/2012 a janeiro/2014. Encontrou-se, entre as principais alegações maternas, dificuldades no processo da amamentação, como mamilos invertidos ou planos, fissuras mamilares e mastites (OLIVEIRA et al., 2015). Esses problemas geralmente ocorrem por falta de preparo das mamas durante a gravidez e puerpério e da técnica inadequada de amamentação (PARIZOTTO et al., 2008). Outros motivos encontrados na presente pesquisa para o desmame precoce foram: doença da mãe $(4,5 \%)$, falta de apoio familiar $(2,1 \%)$ e tabagismo/ alcoolismo (1,1\%).

Quanto à doença da mãe como causa, Araújo et al. (2008) realizaram uma pesquisa qualitativa, em que foram coletados relatos de mães relacionados ao desmame precoce, no qual a maioria declara, dentre outros fatores, que desmamou seus filhos alegando enfermidades associadas geralmente a medicamentos utilizados. Contudo, o estudo afirma que são raras as enfermidades maternas com contraindicação absoluta à amamentação natural, as quais são: portadores de vírus HIV, HTLV 1 E 2, herpes simples nas mamas, moléstias debilitantes graves, desnutrição materna, necessidade de ingestão de medicamentos nocivos à criança por tempo prolongado (ARAÚJO et al., 2008).

Em relação à falta de apoio familiar, o mesmo estudo observou que muitas mães alegaram ter entre as causas do desmame precoce o oferecimento por parte das avós de outro tipo de alimento para o lactante. É dito que essa razão ocorra, possivelmente, pelo fato de avós transmitirem tabus, crenças e proibições inerentes a um dado contexto histórico-social, atuando como elemento desestimulador para a amamentação, considerando-se que a avó é tomada na família como modelo de referência aos cuidados 
com o bebê, inclusive ao aleitamento materno (ARAÚJO et al., 2008).

Por fim, Ciampo et al. (2009) realizaram uma pesquisa com 504 mulheres, das quais 324 (64,3\%) tinham algum tipo de contato com o cigarro. Dentre as 97 mães tabagistas ativas, 78 (80,4\%) afirmaram estar amamentando. 0 estudo encontrou, também, prevalência de $11,1 \%$ de consumo de álcool entre as mães, sendo que destas, $80,3 \%$ estavam amamentando. Assim, observam-se elevadas proporções de mulheres em AME, proporcionando todos os benefícios do aleitamento materno. Porém, as expressivas prevalências de tabagismo e de consumo de bebidas alcoólicas merecem atenção especial e providências no sentido de minimizar ou eliminar esses hábitos nocivos do contexto da saúde materno-infantil.

Pesquisas mostram que o etanol pode alterar o metabolismo dos lipídios e de outros componentes do leite materno, modificando sua composição, valor nutricional e aroma, além de inibir o reflexo da sucção e atenuar o reflexo de ejeção do leite. Já o tabagismo está relacionado a uma menor produção de leite, diminuição da concentração de gordura do leite, redução do tempo de amamentação, além de agredir drasticamente as vias aéreas da lactante e das crianças (CIAMPO et al., 2009).

Desta maneira, ressalta-se a importância das ações educativas no intuito de preconizar a importância do aleitamento materno, as quais devem ser enfatizadas com maior vigor e insistência pelos profissionais de saúde, em todos os níveis de atendimento, para todas as crianças que, por variadas razões, entram no sistema de saúde (ESCOBAR et al., 2002).

Essa necessidade é reforçada pelo fato de o aleitamento materno trazer inúmeros benefícios para o bebê, visto que o desmame precoce é um fator predisponente para doenças evitáveis, como desnutrição, diarreia, obesidade infantil, entre outros problemas de saúde pública no mundo, além de contribuir para o aumento da mortalidade infantil (ALVARENGA et al., 2017). De acordo com a OMS (2014) e o Fundo das Nações Unidas para a Infância (UNICEF), cerca de 6 milhões de crianças não morrem por ano graças à prática de amamentação exclusiva (UNICEF, 2014).

\section{CONCLUSÕES}

Concluiu-se que a prevalência do aleitamento materno exclusivo na pesquisa apresentou um tempo médio abaixo do preconizado pela OMS, além de que o desmame precoce foi ocasionado, principalmente, por mitos e o retorno ao trabalho antes de 6 meses após o parto. A importância do profissional de saúde, incentivando, orientando e apoiando a nutriz durante o acompanhamento pré-natal, a consulta com o pediatra no último trimestre da gestação, o contato pele a pele e amamentação na sala de parto, a observação da mamada no alojamento conjunto, as consultas de puericultura iniciadas na primeira semana de vida, como também o apoio da família e das empresas - garantindo os diretos das mães de amamentarem - são ações que irão reduzir o desmame precoce e, consequentemente, gerar uma menor mortalidade e morbidade infantil, com o aumento da prevalência do aleitamento materno.

\section{REFERÊNCIAS}

ALVARENGA, S. C.; CASTRO, D. S.; LEITE, F. M. C.; BRANDÃO M. A. G.; ZANDONADE, E.; PRIMO, C. C.. Fatores que

influenciam o desmame precoce. Aquichan, v.17, p.93-103,
2017. DOI: http://doi.org/10.5294/aqui.2017.17.1.9

ARAÚJO, O. D.; CUNHA, A. L.; LUSTOSA, L. R.; NERY, I. S.; MENDONÇA, R. C. M.; CAMPELO, S. M. A.. Aleitamento 
materno: fatores que levam ao desmame precoce. Revista Brasileira de Enfermagem, v.6, p.488-492, 2008.

BARROS, F.; HALPERN, R., CESAR, V. G.; TEIXEIRA, A. M. B.; BÉRIA, J. U.. Promoção da amamentação em localidade urbana da região sul do Brasil: estudo de intervenção randomizado. Revista de Saúde Pública, v.28, p.277-283, 1994.

BOCCOLINI, C. S.; BOCCOLINI, P. M. M.; MONTEIRO, F. R.; VENÂNCIO, S. I.; GIUGLIANI, E. R. J.. Tendência de indicadores do aleitamento materno no Brasil em três décadas. Rev Saúde Pública, v.51, n.108, 2017. DOI: https://doi.org/10.11606/S1518-8787.2017051000029

CARVALHO, M. R.; TAMEZ, R. N.. Amamentação: bases científicas para a prática profissional. 2 ed. Rio de Janeiro: Guanabara Koogan, 2002.

CIAMPO, L. A. D.; RICCO, R. G.; FERRAZ, I. S.; DANELUZZI, J. C.; JUNIOR, C. E. M.. Prevalência de tabagismo e consumo de bebida alcoólica em mães de lactentes menores de seis meses de idade. Revista Paulista de Pediatria, v.27, p.361365, 2009. DOI: http://doi.org/10.1590/S010305822009000400003

COTRIM, L. C.; VENÂNCIO, S. I.; ESCUDER, M. M. L.. Uso de chupeta e amamentação em crianças menores de quatro meses no estado de São Paulo. Revista Brasileira de Saúde Materno-Infantil, v.2, p.245-252, 2002. DOI: http://doi.org/10.1590/S1519-38292002000300005

ESCOBAR, A. M. U.; OGAWA, A. R.; HIRATSUKA, M.; KAWASHITA, M. Y.; TERUYA, P. Y.; GRISI, S.; TOMIKAWA, S. O.. Aleitamento materno e condições socioeconômicoculturais: fatores que levam ao desmame precoce. Revista Brasileira de Saúde MaternoInfantil, v.2, p.253-261, 2002. DOI: http://doi.org/10.1590/S1519-38292002000300006
ARQUES, E. S, COTTA, R. M. M.; PRIORE, S. E.. Mitos e crenças sobre o aleitamento materno. Ciência \& Saúde Coletiva, v.16, 2461-2468, 2011.

OLIVEIRA, C. S.; IOCCA, F. A.; CARRIJO, M. L. R.; GARCIA, R. A. T. M.. Amamentação e as intercorrências que contribuem para o desmame precoce. Revista Gaúcha de Enfermagem, v.36, p.16-23, 2015. DOI: http://dx.doi.org/10.1590/19831447.2015.esp.56766

PARIZOTTO, J.; ZORZI, N. T.. Breast-feeding: factors leading to precocious weaning in Passo Fundo, RS. O mundo da Saúde, v.32, p.466-474, 2008.

SAMPAIO, M. A.; FALBO, A. R.; CAMAROTTI, M. C.; VASCONCELOS, M. G. L.; ECHEVERRIA, A.; LIMA, G.. Psicodinâmica interativa mãe-criança e desmame. Psicol: Teor Pesq, v.26, 2010.

SANMAMED, A. F. F.; EUGENIO, L. R.; VRECER, N.. La alfabetización en salud y el empoderamiento de las comunidades. Diálogo igualitário entre los profesionales de la salud y la comunidad. Scripta Nova, v.21, n.17, 2013.

UNICEF. Tracking progress on child and maternal nutrition: a survival and development priority. UNICEF, 2014.

VENANCIO, S. I.; ESCUDER, M. M. L.; SALDIVA, S. R. D. M. GIUGLIANI, E. R. J.. Breastfeeding practice in the Brazilian capital cities and the Federal District: current status and advances. J Pediatr, v.86, p.317-324, 2010. DOI: http://doi.org/10.2223/JPED.2016

VICTORA, C. G.; BEHAGUE, D. P.; BARROS, F. C.; OLINTO, M. T. A.; WEIDERPASS, E.. Pacifier use and short breastfeeding duration: cause, consequence, or coincidence?. Pediatrics, v.99, p.445-53, 1997. DOI: https://doi.org/10.1542/peds.99.3.445

VINHA, V. H. P.. O livro da amamentação. São Paulo: CLR Balieiro, 1999.

A CBPC - Companhia Brasileira de Produção Científica (CNPJ: 11.221.422/0001-03) detém os direitos materiais desta publicação. Os direitos referem-se à publicação do trabalho em qualquer parte do mundo, incluindo os direitos às renovações, expansões e disseminaç̃oses da contribuição, bem como outros direitos subsidiários. Todos os trabalhos publicados eletronicamente poderão posteriormente ser publicados em coletâneas impressas sob coordenação da Sustenere Publishing, da Companhia Brasileira de Produção Científica e seus parceiros autorizados. Os (as) autores (as) preservam os direitos autorais, mas não têm permissão para a publicação da contribuição em outro meio, impresso ou digital, em português ou em tradução. 